\title{
Correction: Calcium plus vitamin D3 supplementation facilitated Fat loss in overweight and obese college students with very-low calcium consumption: a randomized controlled trial
}

Wei Zhu ${ }^{1,2}$, Donglian $\mathrm{Cai}^{2 *}$, Ying Wang ${ }^{2}$, Ning Lin ${ }^{3}$, Qingqing $\mathrm{Hu}^{2}$, Yang $\mathrm{Qi}^{2}$, Shuangshuang $\mathrm{Ma}^{2}$ and Sidath Amarasekara ${ }^{2}$

\section{Correction}

After publication of this article [1], we noted errors to the "body weight" row of Table three. The values for the controls of $\Delta_{1}($ wk $0 \sim$ wk 4$)$ and $\Delta_{2}$ (wk $0 \sim$ wk 8$)$ should be negative (please see Table 1 below, a corrected version of Table three).

Table 1 Body composition changes between baseline and wk 4, wk 8, and wk 12

\begin{tabular}{|c|c|c|c|c|c|c|c|c|c|}
\hline & \multicolumn{3}{|c|}{$\Delta_{1}(w k 0 \sim w k 4)$} & \multicolumn{3}{|c|}{$\Delta_{2}(w k 0 \sim$ wk 8) } & \multicolumn{3}{|c|}{$\Delta_{3}(w k 0 \sim$ wk 12) } \\
\hline & $\begin{array}{c}\text { Calcium+D } \\
(n=26)\end{array}$ & $\begin{array}{l}\text { Control } \\
(n=25)\end{array}$ & $P^{a}$ & $\begin{array}{c}\text { Calcium+D } \\
(n=25)\end{array}$ & $\begin{array}{l}\text { Control } \\
(n=23)\end{array}$ & $P$ & $\begin{array}{c}\text { Calcium+D } \\
(n=22)\end{array}$ & $\begin{array}{l}\text { Control } \\
(n=21)\end{array}$ & $P$ \\
\hline Body weight $(\mathrm{kg})$ & $-2.2 \pm 1.4^{b}$ & $-1.7 \pm 1.2$ & 0.16 & $-3.3 \pm 1.6$ & $-3.3 \pm 1.4$ & 0.94 & $-4.1 \pm 1.8$ & $-3.5 \pm 1.9$ & 0.25 \\
\hline Fat mass $(\mathrm{kg})$ & $-1.7 \pm 1.7$ & $-1.0 \pm 1.1$ & 0.13 & $-2.4 \pm 1.2$ & $-1.7 \pm 0.8$ & 0.03 & $-2.8 \pm 1.3$ & $-1.8 \pm 1.3$ & 0.02 \\
\hline Fat percentage (\%) & $-1.6 \pm 2.5$ & $-0.8 \pm 1.3$ & 0.19 & $-2.1 \pm 1.2$ & $-1.1 \pm 0.9$ & $<0.01$ & $-2.6 \pm 1.6$ & $-1.4 \pm 1.5$ & 0.02 \\
\hline Lean mass (kg) & $-0.4 \pm 1.0$ & $-0.5 \pm 1.2$ & 0.77 & $-0.8 \pm 0.9$ & $-1.2 \pm 1.7$ & 0.24 & $-1.1 \pm 1.1$ & $-1.4 \pm 1.2$ & 0.31 \\
\hline Visceral fat mass $(\mathrm{kg})^{c}$ & $-0.3 \pm 0.3$ & $-0.1 \pm 0.2$ & 0.09 & $-0.4 \pm 0.3$ & $-0.2 \pm 0.2$ & $<0.01$ & $-0.5 \pm 0.2$ & $-0.3 \pm 0.2$ & 0.01 \\
\hline $\operatorname{VFA}\left(\mathrm{cm}^{2}\right)^{c}$ & $-6.6 \pm 7.6$ & $-2.9 \pm 6.6$ & 0.12 & $-10.9 \pm 7.2$ & $-4.6 \pm 4.9$ & $<0.01$ & $-12.0 \pm 6.4$ & $-6.5 \pm 7.2$ & 0.02 \\
\hline
\end{tabular}

Calcium+D, Calcium+vitamin $D_{3}$ group. Control, control group. VFA, visceral fat area.

$\Delta_{1}=[($ mean values wk 4$)-($ mean values wk 0$)] . \Delta_{2}=[($ mean values wk 8$)-($ mean values wk 0$)] . \Delta_{3}=[($ mean values wk 12$)-($ mean values wk 0$)]$.

Received: 5 March 2013 Accepted: 15 March 2013

Published: 8 April 2013

\section{Reference}

1. Zhu W, et al: Calcium plus vitamin D3 supplementation facilitated fat loss in overweight and obese college students with very-low calcium consumption: a randomized controlled trial. Nutr J 2013, 12:8.

\section{doi:10.1186/1475-2891-12-43}

Cite this article as: Zhu et al:: Correction: Calcium plus vitamin D3 supplementation facilitated Fat loss in overweight and obese college students with very-low calcium consumption: a randomized controlled trial. Nutrition Journal 2013 12:43.

\footnotetext{
* Correspondence: nutrition1226@sohu.com

2Department of Clinical Nutrition, Changhai Hospital, Second Military Medical University, Shanghai 200433, China
}

Submit your next manuscript to BioMed Central and take full advantage of:

- Convenient online submission

- Thorough peer review

- No space constraints or color figure charges

- Immediate publication on acceptance

- Inclusion in PubMed, CAS, Scopus and Google Scholar

- Research which is freely available for redistribution

Submit your manuscript at

www.biomedcentral.com/submit

C BioMed Central

\section{() Biomed Central}

(c) 2013 Zhu et al.; licensee BioMed Central Ltd. This is an Open Access article distributed under the terms of the Creative Commons Attribution License (http://creativecommons.org/licenses/by/2.0), which permits unrestricted use, distribution, and reproduction in any medium, provided the original work is properly cited. 